\title{
NECESIDAD DE UNA REFORMA FISCAL MÁS EQUITATIVA EN REPUBLICA DOMINICANA PARA EL AÑO 2022
}

Juan del Rosario Santana ${ }^{1}$

Prof. de la Universidad Autónoma de Santo Domingo (UASD) ORCID 0000-0002-8059-160X juanrosario67@hotmail.com

Para citar este artículo puede utilizar el siguiente formato:

Juan del Rosario Santana: "Necesidad de una reforma fiscal más equitativa en República Dominicana para el año 2022", Revista Caribeña de Ciencias Sociales (vol 10, № 8 octubre-diciembre 2021, pp. 80-96. En línea:

https://doi.org/10.51896/caribe/SERO8745

\section{RESUMEN}

Este artículo tiene como objetivo examinar los factores y aspectos fundamentales de una reforma fiscal progresiva en la Republica Dominicana que grave a las grandes ganancias y que simplifique los procesos burocráticos de tributación. De igual manera se presentan algunas propuestas de aplicación de instrumentos que propicien una mayor equidad y progresividad en momentos en que los ingresos de las clases medias y empobrecidas del país han sido seriamente afectados como resultado de la parálisis del sistema productivo nacional provocado por la pandemia Covid-19. La metodología seguida para este trabajo consistió en un enfoque cualitativo lo que llevó a requerir un estudio descriptivo y comparado del objeto de investigación. Para el logro de estos propósitos, el autor se sustenta en estudios previos y en publicaciones de Organismos Oficiales nacionales e internacionales y publicaciones de expertos en el tema. Las principales conclusiones confirman que la presión tributaria en el país que actualmente es de un 14\% del PIB sigue siendo una de las más bajas de la región de América Latina y Caribe y que los ingresos tributarios descansan fundamentalmente en los impuestos indirectos en un 60.7\% según reporta la DGIl. La conclusión es que resulta muy difícil que el proyecto de reforma fiscal que ha elaborado el gobierno dominicano en este año de 2021 sea progresivo y equitativo y podrá resultar en el pago de más impuestos por parte de los que menos tienen. Además, las nuevas figuras impositivas que se crearían tendrían, en el corto plazo un impacto poco significativo sobre las finanzas públicas. Las mismas podrían ser objeto de cuestionamiento por naciones con las cuales el país tiene acuerdos comerciales, principalmente Estados Unidos de América.

\footnotetext{
1 Director del INISE. Lic. en Economía, Maestrías en Negocios Internacionales con la Universidad de Provence, France, Maestría en Globalización: Procesos Sociales y Políticas Económicas de la Universidad del País Vasco, España, Maestría en Ciencias de la Educación Mención Enseñanza de las Ciencias Económicas y Empresariales, Universidad de Camagüey Cuba.
} 
Palabras clave: Reforma fiscal, Presión Tributaria, Estructura Impositiva, Impuestos Progresivos, Impuestos Regresivos.

\section{NEED FOR A FISCAL REFORM MORE EQUITABLE IN THE DOMINICAN REPUBLIC BY THE YEAR 2022}

\section{ABSTRACT}

The objective of this article is to examine the fundamental factors and aspects of a progressive tax reform in the Dominican Republic that burdens large profits and simplifies bureaucratic taxation processes. In the same way, some proposals for the application of instruments that promote greater equity and progressivity are presented at a time when the income of the country's middle and impoverished classes has been seriously affected because of the paralysis of the national productive system caused by the pandemic Covid 19. To achieve these purposes, the author relies on previous studies and on publications of national and international Official Bodies and publications of experts on the subject. The main conclusions confirm that the tax burden in the country, which currently stands at $14 \%$ of GDP, continues to be one of the lowest in the Latin American and Caribbean region (24\%) (ECLAC 2019) and that tax revenue rests fundamentally on indirect taxes by $60.7 \%$ as reported by the DGII. The conclusion is that it is very difficult for the tax reform project that the Dominican government has drawn up and that it will put to public discussion in this year 2021 to be progressive and equitable and that again, those who have less will pay more taxes. In addition, the new tax figures that would be created would, in the short term, have little significant impact on public finances. They could be questioned by nations with which the country has trade agreements, mainly the United States of America.

Keywords: Tax Reform, Tax Pressure, Tax Structure, Progressive Taxes, Regressive Taxes.

\section{INTRODUCCION}

En el presente trabajo el autor plantea que el aumento excesivo del gasto público para hacer frente a la pandemia Covid-19 y la caída abrupta de los ingresos tributarios como resultado de la parálisis del aparato productivo nacional profundizan la necesidad de que el gobierno dominicano promueva una nueva reforma fiscal en dos direcciones: aumentar los ingresos y por otro lado eficientizar y mejorar la calidad del gasto público. Así este punto de partida permitió al autor caracterizar la actual estructura impositiva dominicana, analizar algunos intentos de reformas fiscales en el año 2020 y en lo que va del 2021 en la región de América Latina y el Caribe, tales como México, Costa Rica y Colombia. Esta última tomada como referencia para sugerir a las autoridades dominicanas mirarse en el espejo colombiano al momento de diseñar la modificación tributaria y así evitar movimientos sociales que pudieran afectar la paz y el clima laboral existentes en el país.

Por otro lado, se examinan algunas propuestas de reforma e instrumentos fiscales de organismos internacionales como la Comisión Económica Para América Latina y el Caribe (CEPAL), el Banco Interamericano de Desarrollo (BID), Fondo Monetario Internacional (FMI) y Banco Mundial (BM) entre otros. 
La metodología seguida para este trabajo consistió en un enfoque cualitativo lo que llevó a requerir un estudio descriptivo y comparado del objeto de investigación, considerando el contexto socioeconómico en el que se desarrolla y las características de la sociedad dominicana. Es decir, considerando la coyuntura de crisis mundial y nacional generada por la pandemia del Covid19 en los últimos dos años. Más específicamente fue utilizada la revisión bibliográfica, donde intentamos establecer posibles respuestas, o acercarnos a las respuestas, a partir de consideraciones más amplias que inciden sobre el fenómeno estudiado. El objetivo fue, por tanto, buscar en las fuentes bibliográficas una forma de responder al problema de investigación. Para la elaboración de los resultados se utilizaron datos económicos de diferentes países y de República Dominicana en particular, considerando diferentes años hasta la actualidad.

Así, el estudio utilizó documentos oficiales, artículos de revistas y periódicos, clasificados como datos secundarios, que engloban la bibliografía ya hecha pública en relación con el tema de estudio, con la finalidad de ponernos en contacto directo con estudios sobre el tema.

\section{ESTRUCTURA DEL SISTEMA TRIBUTARIO DOMINICANO}

La caracterización del actual sistema tributario dominicano permite afirmar que nuestro país continúa siendo una de las economías más dinámicas de la región de América Latina y el Caribe, pero ha visto su Producto Interno Bruto (PIB) crecer en el 2020 a tasas negativas luego de haber crecido sobre el $5 \%$ en promedio en los últimos años. Así su déficit fiscal de alrededor de un $2 \%$ pasó a cerca de un $8 \%$ y su deuda pública ronda aproximadamente el $70 \%$ a marzo del 2021 , luego de registrar niveles cercanos al 50\% al inicio de la pandemia (CEPAL, 2021), por lo que es en este contexto que el gobierno se ha estado planteando la necesidad de una reforma fiscal que contribuya a revertir el déficit fiscal y a generar recursos para hacer frente a las demandas sociales y empresariales del momento.

Para comprender la dimensión de una nueva reforma fiscal y de las figuras impositivas que eventualmente serian modificadas es necesario tener una idea de que como se estructura el actual sistema tributario. En efecto, los impuestos indirectos según la DGII (2018) en el 2015 representaban el $66.4 \%$ de los ingresos tributarios recibidos por el país en ese año y los directos el $33.6 \%$ restante, estructura que es similar al promedio de América Latina, pero diferente al de los países de la Organización para la Cooperación y el Desarrollo Económicos (OCDE). El hecho de que la mayor ponderación corresponda a los impuestos directos indica que, a diferencia de lo que sucede en los países latinoamericanos, los sistemas tributarios de los países más desarrollados tienden a ser más progresivos en la medida en que descansan en una mayor imposición directa (DGIl, 2018). En otras palabras, una reforma fiscal que recaiga sobre los impuestos directos tendrá un efecto más equitativo y progresivo que la que descansa en los impuestos indirectos.

La figura impositiva que más aporta al ingreso tributario del Estado Dominicano es el Impuesto a las Transferencias de Bienes Industrializados y Servicios (ITBIS) que al 2019, aportaba el $47.8 \%$ del total generado. En segundo lugar, estaba el Impuesto Sobre la Renta con un $41.2 \%$, en tercero, el Impuesto a la Propiedad con un 6\%. Comercio Exterior apenas contribuía con un $1.6 \%$, lo 
que evidencia el impacto de la liberalización comercial en este instrumento recaudatorio (Ministerio de Haciendas, 2020).

En la actualidad, las tasas impositivas de los principales impuestos son: Impuesto sobre la Renta a personas físicas son un $25 \%$ de la renta imponible y $27 \%$ para la renta de las empresas y el Impuesto a las Transferencias de Bienes Industrializados y Servicios (ITBIS) es de un $18 \%$ y en algunos casos como las grasas comestibles de un 16\%. En resumen, procedimientos como los anticipos, las retenciones de ITBIS, las tasas diferenciadas, son solo algunos aspectos que dificultan y hacen complejo el sistema tributario dominicano, creando un ambiente propicio para la evasión y elusión de impuestos. (Senior, 2021).

En lo relativo al gasto público, los datos de la ejecución presupuestaria de la nación dominicana al año 2020 muestran que el mismo fue de $\mathrm{RD} \$ 978$ mil millones, de los cuales RD $\$ 865,643.0$ millones (88\%) correspondieron al gasto corriente y el $12 \%$ restante a gastos de capital. En cuanto a la partida corriente, vale apuntar que la misma se incrementó en un $35.0 \%$ en relación con el año 2019, debido a las erogaciones extraordinarias realizadas por el gobierno para los "programas de asistencia social ejecutados en el marco del Plan Económico de Emergencia, para reducir el impacto de la pandemia en la actividad económica y apoyar a los grupos más vulnerables de la sociedad" (Arias, 2021).

La diferencia ingresos menos gastos gubernamentales arrojó un déficit fiscal al 2020 del orden del $7.7 \%$, este comportamiento obedeció a dos razones principales: caída de los ingresos e incremento del gasto público para hacer frente a la enfermedad. Independientemente de la pandemia, lo que confirma que el déficit de la economía dominicana es un problema estructural que produce una necesidad casi constante de introducir reformas fiscales.

\section{Complejidad del Sistema Tributario Dominicano}

En cuanto a la complejidad del sistema tributario dominicano, el mismo estado reconoce que el mismo es complicado sobre todo para Pequeñas y Medianas Empresas (PYMES) por lo que ha creado un Régimen Simplificado de Tributación (RST) el cual es definido por la Dirección General de Impuestos Internos como aquel diseñado para aquellos contribuyentes ya sean personas físicas o jurídicas que cumplan con los requisitos establecidos en el Decreto No. 265-19 con relación a su actividad económica, su nivel de ingresos y sus volúmenes de compras. Este servirá para la determinación del Impuesto Sobre la Renta (ISR) y el Impuesto sobre Transferencias de Bienes Industrializados y Servicios (ITBIS), basado en sus ingresos brutos o en sus compras.

De acuerdo con el artículo 2 del Decreto 265-19 el ámbito de aplicación del régimen incluye a personas físicas o jurídicas prestadores de servicios o pertenecientes al sector agropecuario, personas jurídicas productoras de bienes y a personas físicas o jurídicas comerciantes de bienes. Esto abarca ambas modalidades es decir de ingresos como de compras. En el caso de la modalidad de ingresos, el decreto consigna que solamente podrán acogerse aquellas personas o entidades cuyos ingresos brutos anuales no superen los ocho millones setecientos mil pesos ( $\operatorname{RD} \$ 8,700,000)$. Asimismo, establece que la modalidad de compras solo aplica para aquellos contribuyentes cuyas compras e importaciones totales no superen los cuarenta millones de pesos ( $\operatorname{RD} \$ 40,000,000$ ) 
dominicanos para cada ejercicio fiscal. Este monto será ajustado anualmente por la inflación. (Decreto 265-19, articulo 5). El artículo 6 del decreto antes citado, establece que solamente pueden aplicar al RST aquellos contribuyentes que cumplan con los siguientes requisitos:

a) Estar inscritos en el Registro Nacional de Contribuyentes (RNC)

b) Tener actualizado a la fecha de presentación de solicitud, sus datos del RNC. Si es una persona jurídica deben estar actualizado las informaciones sobre el monto del capital, generales de los accionistas y beneficiario final, actividad económica y tipo societario, así como cualquier otro cambio. Además, contar como cierre fiscal el 31 de diciembre, estar al día en el cumplimiento de sus obligaciones tributarias y deberes al momento de la solicitud y no encontrarse en proceso de fiscalización externa.

Datos publicados por la Dirección General de Impuestos Internos (DGII, 2020a) dan cuenta que el año 2019 estaban registradas en esa dirección unas 234,498 empresas contribuyentes de las cuales las MIPYMES representaban el $96.1 \%$, mientras que el $3.9 \%$ restante corresponde a las clasificadas como grandes empresas. Asimismo, de las 224,740 MIPYMES registradas en la DGII, la mayor parte eran microempresas (86.9\%), seguidas de las pequeñas $(12.5 \%)$ y en la tercera posición de las medianas $(0.5 \%)$. Esto demuestra que no solo las MIPYMES aportan gran parte de los empleos generados sino también de los ingresos tributarios, aunque ha de tomarse en cuenta que las grandes pueden ser las menos, pero las que más contribuyan por su volumen de operaciones.

Al analizar la empresa según tipo de persona, la entidad recaudadora antes citada reporta que al año 2019 el 55.7\% de las MIPYMES estaban constituidas bajo personas físicas y el $44.3 \%$ jurídica y el $85.1 \%$ tenían como actividades económicas a los servicios, $11.3 \%$ a industrias y solamente el $3.6 \%$ a las agropecuarias. En cuanto al recaudo efectivo de las MIPYMES, este organismo consigna que para el periodo 2012-2019 las mismas aportaron el 9.4\% del recaudo efectivo de la DGIl, el cual tan solo en el año 2019 fue de 49,827.3 millones de pesos dominicanos. En lo relacionado con las recaudaciones de las MIPYMES, el organismo oficial sostiene que el $51.9 \%$ del total recaudado por la Dirección General de Impuestos Internos (DGII) durante el intervalo 2012-2019 corresponde a las figuras impositivas del Impuesto Sobre la Renta a Personas Físicas, Jurídicas y Asalariados y del Impuesto sobre la Transferencia de Bienes Industrializados y Servicios (ITBIS).

Otro elemento que destaca el organismo recaudador es que el impuesto sobre la renta de personas jurídicas supera casi tres veces al de personas físicas. Similar comportamiento se muestra en las operaciones con el Impuesto a las Transferencias de Bienes y Servicios (ITBIS). También el boletín de la DGII (2020르) recoge que el número de empresas MIPYMES que tributaban al año 2019 bajo el Régimen Simplificado de Tributación (RST) era de 11,151 MiPymes, de las cuales el $90.7 \%$ se encontraban acogidas al RST de ingresos, mientras que el restante $9.3 \%$ al de compras. Esto equivale al $46.8 \%$ del total de contribuyentes acogidos al RST y confirma que la mayor proporción de los contribuyentes acogidos al RST son microempresas (86.1\%).

El trabajo de investigación publicado en el periódico El Día por la periodista Silvestre (2020) cita como una de las ventajas de este régimen el hecho de que las empresas o personas físicas que se acogen no tienen que pagar anticipos del Impuesto Sobre la Renta, así como impuestos a los 
activos relacionados con actividades económicas mencionadas. Además, elimina la obligatoriedad de enviar mensualmente los formularios de datos establecidos por la DGll para el régimen ordinario y dispone de una sola declaración anual automática, pero no aplica saldo a favor a deudas con la DGII. Sin embargo, continúan obligados a emitir facturas con Comprobante Fiscales y requerir facturas con valor fiscal a sus proveedores para fines informativos, ya que no podrán utilizar dichas facturas para fines de deducción o adelanto de costos y gastos. Representantes de organizaciones de comerciantes y empresarios organizados en la Federación Dominicana de Comerciantes y Empresarios de la República Dominicana (FENACERD) sugieren al gobierno como paso para facilitar la incorporación de las micro, pequeñas y medianas empresas (Mipymes) al RST la aplicación de una Amnistía Fiscal que permita que ingresen al sistema sin trauma y sin objeciones.

En un trabajo realizado por los investigadores Zatsman y Zentner (2021), plantearon que la complejidad del sistema impositivo dominicano dificulta el combate de la evasión y la elusión de las obligaciones tributarias por lo que se hace necesario diseñar formas simples de tributación.

\section{Gasto tributario y evasión fiscal en la República Dominicana}

Otros aspectos claves que caracterizan la actual estructura del sistema tributario de la República Dominicana son el gasto tributario y la evasión fiscal, entendido el primero como todas aquellas exenciones y subvenciones que otorga el gobierno dominicano a diversos sectores de la economía y el segundo como el no pago consciente de algún impuesto establecido por las leyes del pais. En el caso del gasto tributario, los datos publicados por el Ministerio de Haciendas de la República Dominicana (2020) y recogidos en el cuadro 1, muestran que mediante esta figura el gobierno dominicano se propone transferir en el año 2021, alrededor de unos RD $\$ 209,657.1$ millones equivalentes al $4.28 \%$ del Producto Interno Bruto del país. De esa suma, el 55\% corresponde a exenciones del Impuesto a la Transferencia de Bienes Industrializados y Servicios (ITBIS), un $16 \%$ al Impuesto Sobre la Renta y un $13 \%$ al Impuesto al Patrimonio. Las exoneraciones y exenciones por concepto de ISC Hidrocarburos representaran el $8 \%$ y las del arancel el $6 \%$. Los otros porcentajes se distribuyen entre otros ISC e impuestos sobre el uso de bienes y licencias. 


\section{Cuadro 1}

Estimación del Gasto Tributario, año 2021

\begin{tabular}{|l|r|r|r|}
\hline \multicolumn{1}{|c|}{ IMPUESTO } & MILLONES RDS & $\begin{array}{c}\text { PORCENTAJE } \\
\text { DEL PIB }\end{array}$ & $\begin{array}{l}\text { PARTICIPACION \% } \\
\text { DEL TOTAL }\end{array}$ \\
\hline ITBIS & $116,220.50$ & $2.37 \%$ & $55 \%$ \\
\hline ISR & $32,874.00$ & $0.67 \%$ & $16 \%$ \\
\hline Impuesto al Patrimonio & $27,562.10$ & $0.56 \%$ & $13 \%$ \\
\hline ISC Hidrocarburos & $16,017.00$ & $0.33 \%$ & $8 \%$ \\
\hline Arancel & $11,821.70$ & $0.24 \%$ & $6 \%$ \\
\hline Otros ISC & $4,551.70$ & $0.09 \%$ & $2 \%$ \\
\hline Impuesto sobre el uso de & & & \\
\hline bienes y licencias & 610.1 & $0.01 \%$ & $0 \%$ \\
\hline TOTAL & $209,657.10$ & $4.27 \%$ & $100 \%$ \\
\hline
\end{tabular}

Fuente: Ministerio de Haciendas (2020).

En lo relativo a la evasión fiscal, datos publicados por el Ministerio de Hacienda de la República Dominicana (2018) dan cuenta de que esta aún permanece elevada. Cita, por ejemplo, que la evasión del ITBIS en el país ronda el $43 \%$ y por cada punto de evasión el estado deja de recibir alrededor de 4,000 millones de pesos, es decir, aproximadamente unos RD\$172 mil millones, equivalente al $4 \%$ del Producto Interno Bruto de la República Dominicana. Luego, está la del Impuesto Sobre la Renta que se aproxima al 60\% principalmente en el componente de personas jurídicas que es el más elevado (empresas) y esto significa que por cada 1\% porcentual de evasión, el Estado registra una merma de 2 mil millones de pesos, lo que equivaldría entonces 120 mil millones de pesos, un $3 \%$ aproximado del PIB.

El cuadro 2 recoge los datos publicados por el Ministerio de Hacienda (2018) y la Dirección General de Impuestos Internos (2020) que permiten inferir que por concepto de gasto tributario y evasión fiscal el gobierno dominicano dejó de recibir en el 2019 alrededor de RD\$533 mil millones, un $11.69 \%$ del Producto Interno Bruto (PIB), de los cuales un $7.05 \%$ corresponde a gasto tributario y el $4.94 \%$ restante a evasión fiscal. De esta última, los datos oficiales indican que la evasión del ITBIS en el país ronda el $43 \%$ del que según las autoridades se debió cobrar por este impuesto, seguido 
del Impuesto Sobre la Renta la cual se aproxima al 60\% principalmente en el componente de personas jurídicas que es el más elevado (empresas).

\section{Cuadro 2}

Porcentaje de evasión y gasto tributario en monto y porcentaje del PIB 2019

EN MILLONES DE RD\$

\begin{tabular}{|c|c|c|c|c|}
\hline \multirow{2}{*}{ FIGURA } & \multirow{2}{*}{$\begin{array}{c}\text { TOTAL } \\
\text { RECAUDADO (2019) }\end{array}$} & \multicolumn{2}{|c|}{ INCUMPLIMIENTO } & \multirow{2}{*}{ EVASION \% PIB } \\
\hline & & Millones RD\$ & incumplimiento & \\
\hline Evasión del ITBIS & $120,581.97$ & $89,712.99$ & $43.60 \%$ & $1.97 \%$ \\
\hline Evasión del ISR Empresas & $96,183.94$ & $141,967.50$ & $61.90 \%$ & $3.11 \%$ \\
\hline Evasión del ISR Personas & $59,452.96$ & $76,337.60$ & $57.10 \%$ & $1.67 \%$ \\
\hline Subtotal incumplimiento fiscal & $276,218.87$ & $308,018.08$ & & \\
\hline & $225,485.10$ & $225,485.10$ & & $4.94 \%$ \\
\hline Total sacrificio fiscal & & $533,503.18$ & & $11.69 \%$ \\
\hline
\end{tabular}

Fuente: Ministerio de Haciendas (2018) y la Dirección General de Impuestos Internos(2020).

Cardoza (2018) citando a Fenochietto en un informe elaborado para el Fondo Monetario Internacional resume las características del sistema tributario dominicano de la manera siguiente: "se ha creado así un régimen tributario complejo, inestable (seis reformas en 9 años) y difícil de controlar, que no cumple con ninguno de los principios básicos de tributación: suficiencia (para financiar un gasto público mínimo); equidad (que los habitantes de un país contribuyan a las arcas del Estado en la medida de su capacidad) y eficiencia (que el régimen altere mínimamente las decisiones de los productores y consumidores)". Esto lo hace complejo y costoso tanto para el contribuyente como para la Administración Tributaria. Concluye el autor que existe una gran oportunidad para la futura discusión del Pacto Fiscal donde uno de los principales puntos de discusión debería estar enfocado en la simplificación del Sistema Tributario con el fin de reducir los costos de cumplimiento y la evasión fiscal tal como lo dicta la Estrategia Nacional de Desarrollo.

\section{LAS REFORMAS FISCALES IMPLEMENTADAS EN AMERICA LATINA Y LA REPÚBLICA DOMINICANA EN EL CONTEXTO DE LA PANDEMIA COVID-19}

En la región de América Latina, algunos países se han estado planteando reformas fiscales que persiguen mejorar las finanzas públicas erosionadas por los gastos incurridos para hacer frente a los efectos sanitarios y económicos provocados por la pandemia Covid-19. Según señala Dannemann (2021), estas modificaciones persiguen gravar a las personas y empresas con mayores ingresos, revirtiendo así el actual esquema en el que 10\% de las personas con mayores ingresos en Latinoamérica pagan una tasa impositiva efectiva promedio de solo el 5,6\% sobre sus ingresos, en algunos casos solo de $1 \%$ a $3 \%$. A partir de esta realidad el autor sugiere que es necesario avanzar en una reforma tributaria que grave los ingresos y patrimonios más elevados, coinciden los expertos. "En el pasado casi todas las reformas han aumentado los impuestos indirectos (al consumo, como el IVA). De hecho, la mitad de la recaudación de la región viene de impuestos indirectos y falta 
tributación de la riqueza"." No hay un control o una fuerza impositiva del estado sobre las grandes riquezas de estas personas. No están declarando casi nada", agrega.

En el debate sobre el tipo de reformas fiscales que requiere la economía de la región de América Latina y el Caribe para reducir déficits y endeudamiento, el Banco Interamericano de Desarrollo plantea que estos "necesitan urgentemente implementar reformas fiscales para preparar el terreno para una recuperación sustentable post Covid" (BID, párr. 1, 2021). Afirma que

los sistemas fiscales saludables pueden ayudar también a liberar nuestro potencial, apalancando la reasignación de recursos en todos los sectores y así impulsar el crecimiento de la productividad, promover el empleo formal, y lograr un futuro verde que desafíe la falsa dicotomía entre crecimiento y sostenibilidad ambiental (BID, párr. 7, 2021).

Por otro lado, la Comisión Económica Para América Latina y el Caribe, CEPAL (2021) ha planteado que

será necesario que en la política fiscal se adopte una perspectiva estratégica en que se promuevan acciones de gasto público enfocadas en crear sistemas de protección social universales e incentivar inversiones intensivas en empleo que sean ambientalmente sostenibles y estén dirigidas a sectores estratégicos. También se requerirá una política tributaria progresiva que contribuya a reducir las desigualdades de ingreso y a financiar el gasto público (CEPAL, p. 48, 2021).

Para este organismo,

dar sostenibilidad a una política fiscal expansiva requiere generar ingresos fiscales que permitan financiar las acciones de gasto público. Sin embargo, históricamente, los ingresos públicos de América Latina y el Caribe han sido insuficientes para atender las demandas del gasto público, lo que ha dado lugar a un sesgo deficitario, a una tendencia procíclica de la política fiscal (CEPAL, $p$. $51,2021)$

y a una presión tributaria baja. Entiende la CEPAL que generar más ingresos es una de las tareas más prioritarias de nuestros países para que los sistemas tributarios puedan estar al servicio de los Objetivos de Desarrollo Sostenible (ODS). Las estrategias que deben implementarse para aumentar los ingresos fiscales son entonces: Reducir los espacios para la evasión y la elusión tributarias, evaluar el uso de los gastos tributarios y los incentivos fiscales, y orientarlos hacia los ODS, reforzar el impuesto sobre la renta de las personas físicas, ampliar el alcance de los impuestos sobre los bienes inmuebles y el patrimonio y aplicar impuestos a la economía digital e impuestos correctivos como los impuestos verdes y los relacionados con la salud pública, y fortalecer los que ya existen (CEPAL, pp. 51-53, 2021). 
Según el organismo multilateral antes citado plantea "para que los países de América Latina se encaminen hacia sistemas tributarios progresivos es imprescindible fortalecer los impuestos directos, en particular el impuesto sobre la renta de las personas físicas" (CEPAL, p. 68, 2021).

Al revisar algunos intentos de reformas fiscales en países de la región se observa que a pesar de los esfuerzos, estas no trascienden hacia la progresividad por lo que de implementarse podrían afectar a las clases de menores ingresos. Por ejemplo, en Costa Rica, el Ministerio de Haciendas de Costa Rica (2020), establecía entre otras medidas de su reforma, la reducción del gasto tributario y la creación de impuestos temporales y extraordinarios. En cuanto a la propuesta del gobierno de Costa Rica sobre una reforma fiscal, esta consistía en gravar la renta de personas que realizaban actividades consideradas independientes, no aumentar salarios del sector público hasta reducir el topo de deuda por debajo del $60 \%$ del PIB, implementar el Impuesto al Valor Agregado (IVA) a los Servicios Digitales Transfronterizos, gravar las ganancias de capital previa indexación del valor de adquisición, como ocurre con la legislación vigente. En el caso del gasto, el gobierno se propone además reducir y contener el gasto público y otras medidas fiscales temporales serían crear un impuesto extraordinario sobre la renta, gravar las remesas al exterior en un $5 \%$ y crear un impuesto a las transacciones de pagos electrónicos y cheques. Con estas medidas Costa Rica persigue impactar el PIB en un 6\% (Ministerio de Haciendas de Costa Rica, 2020).

En cuanto la propuesta mexicana, se modifica el Impuesto Sobre la Renta y el Impuesto al Valor Agregado principalmente. Según Hubard (2021), los elementos básicos que permitirían lograr una reforma fiscal eficiente deberían entre otras cosas: Aumentar el IVA a niveles del $20 \%$, quitando las exenciones y solo eximirlo a las zonas de mayor pobreza en el país, reducir del $30 \%$ al 20 o $22 \%$ la tasa del ISR, lo que posiciona a México como un país muy atractivo para la generación de empresas nuevas; que las actuales aumenten su inversión; que llegue un importante flujo de inversión extranjera directa para generar empleos, mayor flujo de dinero en la economía, mayor consumo y por lo mismo, mayor recaudación.

En cuanto a la reforma presentada en abril 2021 por Colombia y que debido a las presiones sociales tuvo que ser retirada y aplazada se afirma que la misma planteaba colocar impuestos a productos básicos de la canasta familiar, aumentar el costo de la gasolina, ampliar la base gravable del impuesto de renta, imponer un impuesto solidario para salarios altos, poner impuesto al patrimonio por dos años, IVA para los servicios funerarios y los servicios de internet, entre otros" (Ministerio de Haciendas de Colombia, 2021).

Con ella el gobierno colombiano pretendía recaudar a partir del 2022 , el equivalente al $2 \%$ del PIB en nuevos impuestos, es decir, unos 23 billones de pesos colombianos adicionales (unos US\$6.300 millones de dólares) para enfrentar la crisis económica en la que está sumido el país, muy afectado por la pandemia de coronavirus. Se observa que era una reforma con carácter altamente regresivo por lo que encontró rápidamente el rechazo de la población y tuvo que ser retirada debido a las presiones sociales generadas. 
En otros países de la región se han estado diseñando reformas fiscales con figuras impositivas según Toro (2021) cuyos debates giran en torno al incremento del impuesto a la riqueza. Sugiere el autor que en países como Argentina el debate se centra en la efectividad que pudiera tener un cobro único de entre 2,25\% y 5,25\% para quienes declaren ganancias anuales superiores a US $\$ 2,2$ millones. El impuesto que, sería conocido como "contribución extraordinaria", no ha mostrado efectividad. En el caso de Chile, el impuesto sería de 2,5\% y afectaría a quienes tengan patrimonios superiores a los US $\$ 22$ millones. Citó que Estados Unidos se propone un paquete fiscal integrado por tres pilares con el cual se intenta captar unos 2.5 billones de dólares durante 15 años: mediante el aumento a $28 \%$ del impuesto a sociedades, la imposición de medidas a empresas que tributan en otros países con climas fiscales más favorables y la eliminación de subsidios a empresas de combustibles fósiles, mientras los reemplaza por bonos para la producción de energías limpias.

La revisión a reformas fiscales implementadas en la República Dominicana arroja que las mismas han estado sustentadas en una tributación regresiva, cuyas principales figuras impositivas han sido la ampliación de la base y tasas del Impuesto a las Transferencias de Bienes y Servicios (ITBIS), modificación de las tasas del Impuesto Sobre la Renta y los Impuestos a la Propiedad entre otros. Este comportamiento fue reiterado en el fallido intento de reforma tributaria que las autoridades rectoras de las finanzas públicas del país sometieron al Congreso Nacional para su aprobación en el presupuesto del 2021. En esta se planteaba entre otras, el establecimiento de un impuesto a las ganancias extraordinarias de empresas con un 8\%, nuevos gravámenes al Gas Licuado de Petróleo (GLP), ampliación de la base impositiva y aumento de tasas para los bancos comerciales, el gravamen con $3 \%$ de la compra de dólares, imposición a los servicios digitales y una mayor imposición a personas físicas. Además, gravar el salario 13 de los trabajadores dominicanos. Este intento, encontró el rechazo de amplios sectores de la población dominicana, quienes entendían que no era el momento y el equipo económico tuvo que retroceder.

En un artículo periodístico publicado por Freund (2021) planteaba que se ha estado hablando de que una reforma fiscal en la República Dominicana incluiría un paquete de impuestos en los que se contemplaría entre otras cosas, la disminución en la tasa de impuestos sobre los bienes y servicios industrializados y en contraprestación habría una ampliación del gravamen (ITBIS) en la cantidad de bienes y servicios industrializados que alcanzaría, con la excusa de mitigar la inflación originada por la pandemia del coronavirus, sin embargo, esto juega a ser un arma de doble filo. En esta misma dirección, Reyes (2021) establece que para afianzar la equidad, eficiencia y sostenibilidad de las finanzas públicas, el país debería mejorar la gestión administrativa, reforzar la infraestructura tecnológica, reducir los gastos fiscales, reformar el ITBIS, eliminando exenciones y simplificando las tasas, reducir el Impuesto Sobre la Renta, ampliar la base imponible del impuesto sobre los activos y racionalizar los incentivos tributarios entre otras medidas.

\section{CONCLUSIONES Y RECOMENDACIONES}

- La República Dominicana ha estado sometida a una presión constante de búsqueda de ingresos para cubrir déficits, lo cual se convierte en una búsqueda contantes de alternativas 
generadoras de ingresos, principalmente de reformas que solamente afectan los ingresos tributarios y no el gasto.

- Cada vez que el país presenta una reforma fiscal, genera reacciones negativas en la población, debido a que la población no ve calidad en los servicios públicos y percibe que los nuevos recaudos irán a parar a los bolsillos de funcionarios, políticos y grandes empresarios.

- La estructura del sistema tributario dominicano es compleja a pesar de las reformas y normas administrativas implementadas, las cuales han servido más para crear y aumentar impuestos que para hacerlo más simple y eficiente.

- La existencia de varias leyes de incentivos que coexisten con el sistema tributario actual, de un gasto tributario excesivo, de una baja recaudación lo hace deficiente y costoso tanto para el Estado como para el contribuyente.

- El gasto tributario, la evasión fiscal y la existencia de múltiples leyes, tasas y procesos hacen la tributación dominicana compleja y costosa, lo cual reafirma la existencia de una amplia brecha fiscal en el actual sistema tributario dominicano.

- A igual que en la reforma tributaria del 1992 ha de esperarse que la del 2021 sea el comienzo de una ola de modificaciones impositivas con el deliberado propósito de captar más ingresos para financiar el gasto público.

- De las reformas fiscales planteadas en la Región de América Latina que fueron presentadas sucintamente en este artículo, la de Costa Rica parecería ser la más progresiva y equitativa, mientras que la de Colombia sería la más regresiva por lo que el gobierno dominicano debería verse en el espejo de los colombianos al momento de diseñar su propuesta.

- Es probable que la nueva reforma fiscal propuesta por el gobierno dominicano esté sustentada nueva vez en impuestos indirectos, de por sí, con un carácter regresivo, en el que nueva vez, pagaran más impuestos lo que menos tienen. Esto se evidencia en la dilación que ha tenido el gobierno en someter su discusión ante el Consejo de Desarrollo Económico y Social.

- La reforma fiscal que proponga el gobierno debería al menos contener medidas para eficientizar el gasto, reducir la evasión fiscal y el gasto tributario y la misma deberá ser elaborada con visión integral en la que el pacto eléctrico, la reforma al sistema de seguridad social y la reforma laboral sean parte también de las discusiones.

- La reforma fiscal que se apruebe deberá estar acompañada de normas que viabilicen la tributación y que reduzcan a su mínima expresión la visión de organismo de persecución fiscal de la DGII. 
- Una reforma fiscal que solo persiga la captación de ingresos al Estado podría afectar negativamente la competitividad empresarial, el ritmo de crecimiento de la economía, la inversión local y extranjera, el empleo y la formalidad tanto en el trabajo como en las empresas.

- Si se quiere una reforma fiscal más equilibrada y en consonancia con lo que se discute en la región de América Latina deberá apoyarse en tres pilares: Grabar a las grandes ganancias y sociedades, revisión del gasto tributario o subsidios a sectores económicos y la reducción de la evasión fiscal y la aplicación de un sistema simplificado y efectivo para las PYMES cuyo propósito debería ser la formalización y no la captación de ingresos, al menos en un primer momento.

- Cualquier intento de aplicación de reforma fiscal en el país deberá ser el resultado de un amplio consenso social en el que los diferentes sectores económicos y sociales se sientan verdaderamente representados. Por tanto, universidades, sindicatos, ONGs de la Sociedad Civil, grupos sindicales y empresariales deberán tener una participación en el espacio creado para estos fines.

\section{REFERENCIAS}

Arias, Socorro (05/03/2021): El Gobierno de RD pagó RD\$122,632.3 millones en programas sociales por COVID-19. Diario Libre. https://www.diariolibre.com/economia/el-gobierno-de-rd-pago-rd1226323-millones-en-programas-sociales-por-covid-19-DI24792463. En línea al 21 de mayo 2021.

Banco Interamericano de Desarrollo (BID, 2020): BIDeconomics República Dominicana, Oportunidades para un desarrollo sostenible, inclusivo y resiliente. Grupo BID, República Dominicana. 2020.

Banco Interamericano de Desarrollo (BID, 2021a): Costa Rica avanzará en su reforma fiscal para promover el crecimiento con apoyo del BID. Comunicado de Prensa. Costa Rica, 5 de mayo del 2021. https://www.iadb.org/es/noticias/costa-rica-avanzara-en-su-reforma-fiscal-parapromover-el-crecimiento-con-apoyo-del-bid. En línea al 30 de mayo 2021.

Banco Interamericano de Desarrollo (BID, 2021): Informe macroeconómico BID: reformas fiscales clave para recuperación post pandemia. Comunicado de Prensa. Colombia, marzo, 20, 2021. https://www.iadb.org/es/noticias/informe-macroeconomico-bid-reformas-fiscales-clave-pararecuperacion-post-pandemia. En línea al 30 de mayo 2021. 
Banco Central de la República Dominicana (2021): Informe de la Economía Dominicana: enerodiciembre 2020". Santo Domingo, República Dominicana. Marzo 2021.

Banco Central de la República Dominicana (2020): Resultados preliminares de la actividad económica enero-abril 2020. índice Mensual de Actividad Económica (IMAE). República Dominicana. Mayo 2020.

Bosch Carcuro, Matías y Fernández Gil, Airon (2020): Del Negocio Seguro a la Seguridad Social. Colección La verdad en manos de los buenos. Fundación Juan Bosch. República Dominicana.

Cardoza, Marvin (2018): Sistema tributario de RD: Análisis de sus características y complejidades. Periódico El Dinero, República Dominicana. 22 de agosto del 2018.

CEPAL (2021). Panorama Fiscal de América Latina y el Caribe. https://www.cepal.org/sites/default/files/publication/files/46808/S2100170_es.pdf. En línea al 22 de septiembre de 2021.

Cota, Isabella (2021): La reforma tributaria de Colombia incluye una renta básica. Periódico El País, sección Economía. 16 de abril del 2021. Colombia.

Dannemann, Victoria (2021): América Latina y Covid: Argumentos para un impuesto a la riqueza. DW Made For Mind, América Latina, 1 de mayo del 2021. https://www.dw.com/es/empresa/publicidad/s-48074235. En línea al 5 de junio 2021.

Del Rosario Santana Juan (2020): Incidencia del Covid-19 en el Empleo de la República Dominicana. Elaborado por el Instituto de Investigación Socioeconómica de la Facultad de Ciencias Económicas y Sociales de la Universidad Autónoma de Santo Domingo. República Dominicana. Mayo 2020.

Dirección General de Crédito Público (2021): Deuda del Sector Público No Financiero al 2019 y 2020. Ministerio de Hacienda de la República Dominicana. Mes de abril 2021. https://www.creditopublico.gob.do/. En línea al 18 de mayo del 2021.

Dirección General de Impuestos Internos [DGII] (2018): Sistema Tributario de la República Dominicana. Agosto del 2018. https://dgii.gov.do/publicacionesOficiales/estudios/Documents/2018/Sistema\%20Tributario\%2 0de\%20la\%20Rep\%C3\%BAblica\%20Dominicana.pdf. En línea al 18 de mayo 2021. 
Dirección General de Impuestos Internos [DGII] (2020a): Boletín MIPYMES 2020. Elaborado por la Gerencia de Estudios Económicos y Tributarios. República Dominicana. Noviembre del 2020. https://dgii.gov.do/estadisticas/Boletin\%20Mipymes/Boletin-MIPYMES-2020.pdf. En línea 18 de mayo 2021.

Dirección General de Impuestos Internos [DGII] (2020b) Memoria Anual 2019. República Dominicana.

https://dgii.gov.do/transparencia/planEstrategico/memoriasInstitucionales/Documents/Informe Gestion19.pdf. En línea al 18 de mayo de 2021.

Freund, Ignatz (26-04-2021): Hacia una nueva reforma fiscal. Periódico Digital Acento. https://acento.com.do/el-financiero/hacia-una-nueva-reforma-fiscal-8937879.html. En línea al 27 de mayo del 2021.

García, Sudelka (2021): EE.UU Celebra Pacto Sin Precedentes para un Impuesto a Multinacionales. Periódico El Nacional, sección Economía. República Dominicana. 5 de junio 2021. https://elnacional.com.do/eeuu-celebra-pacto-sin-precedentes-para-un-impuesto-amultinacionales/. En línea al 5 de junio del 2021.

Hubard, Mauricio (2021): México sí necesita una reforma fiscal, pero a fondo. Artículo publicado en la Revista Expansión, sección Opinión de fecha 13 de marzo del 2021 . México. https://expansion.mx/opinion/2021/03/12/mexico-si-necesita-una-reforma-fiscal-pero-a-fondo. En línea al 30 de mayo del 2021.

Instituto de Investigación Socioeconómica de la Facultad de Ciencias Económicas y Sociales de la Universidad Autónoma de Santo Domingo (INISE, 2020): Análisis de Coyuntura sobre la Incidencia del Covid-19 en el Empleo de la República Dominicana: mayo 2020.

La Organización para la Cooperación y el Desarrollo Económicos y otros (OCDE, 2020): Estadísticas tributarias en América Latina y el Caribe 2020 - Costa Rica.

López Pérez, Sugey de J. y Vence, Xavier (2021): Estructura y evolución de ingresos tributarios y beneficios fiscales en México. Análisis del periodo 1990-2019 y evaluación de la reforma fiscal de 2014. Revista Trimestre Económico. 2 de mayo del 2021. https://www.eltrimestreeconomico.com.mx/index.php/te/article/view/1104/1287. En línea al 30 de mayo del 2021.

Ministerio de Economía, Planificación y Desarrollo (MEPYD, 2018): El envejecimiento de la población y el sistema de seguridad social. República Dominicana. 3 de diciembre del 2018. 
Ministerio de Haciendas de Colombia (2021): Medidas Covid-19. https://www.minhacienda.gov.co/webcenter/portal/Minhacienda. En línea al 21 de mayo 2021.

Ministerio de Haciendas de Costa Rica (2020): Plan para superar el impacto fiscal de la pandemia. Costa Rica, 17 de septiembre del 2020.

Ministerio de Haciendas de la República Dominicana. (2018): Estimación del Incumplimiento Tributario en la República Dominicana. República Dominicana, octubre del 2018. https://dgii.gov.do/publicacionesOficiales/estudios/Documents/2018/IncumplimientoTributario-en-RD.pdf. En línea al 29 mayo 2021.

Ministerio de Haciendas de la República Dominicana (2020): El Gasto Tributario en República Dominicana 2021. Elaborado para la Comisión Interinstitucional Coordinada por la Dirección General de Política y Legislación Tributaria". Septiembre del 2020. https://www.hacienda.gob.do/wp-content/uploads/2021/01/2020-10-16-Estimacion-del-GastoTributario-2021.pdf. En línea al 21 de mayo 2021.

Organización Panamericana de la Salud (OPS), Organización Mundial de Salud (OMS) y Consejo de Instituciones de Seguridad Social de Centroamérica y República Dominicana (CISSCAD) (2020): Seguridad Social en Centroamérica y República Dominicana: Situación actual y desafíos. Washington, D.C, Estados Unidos de Norteamérica. Año 2020.

Periódico El Día (2021): "Crédito privado en moneda nacional sube RD\$91 mil millones en últimos 12 meses". Sección Económica de fecha 21 de mayo del 2021. República Dominicana. https://eldia.com.do/credito-privado-en-moneda-nacional-sube-rd91-mil-millones-en-ultimos12-meses/. En línea al 21 de mayo 2021.

Quesada, Juan Diego (2021): Duque cede a las protestas y retira la reforma tributaria de Colombia. Periódico El País, sección Internacional. 2 de mayo 2021. Bogotá, Colombia. https://elpais.com/internacional/2021-05-02/el-presidente-de-colombia-retira-la-reformatributaria.html. En línea al 21 de mayo 2021.

Reyes Rodríguez, Patria (2021): ¿Sabes lo que propone el Banco Interamericano de Desarrollo para mejorar las finanzas públicas? Artículo publicado en el periódico Listín Diario. Sección Economía y Negocios. 10 de junio del 2021. 
Senior Fernández, Jaime (29-42021): ¿Es hora de realizar la reforma fiscal? Diario El Dinero. https://eldinero.com.do/158206/es-hora-de-realizar-la-reforma-fiscal/. En línea al 21 de mayo 2021.

Silvestre, Senabri (2020): Nuevo Régimen ha Captado 13,456 Contribuyentes. Periódico El Día, sección Económica. República Dominicana. 2 de enero del 2020.

Severino, Jairo (2021): La deuda dominicana paso de perspectiva estable a negativa. Artículo publicado en el periódico El Dinero, República Dominicana. 11 de marzo del 2021. En línea al 20 de mayo del 2021.

Tejero Puntes, Suhelis (2021): República Dominicana ya adeuda $70.5 \%$ de su PIB. La deuda superó los 58,000 millones de dólares hasta marzo. Periódico Diario Libre. Sección Económica, Santo Domingo, República Dominicana. 26 de mayo del 2021.

Tesorería de la Seguridad Social (2020): Boletín Mensual presentado por la TSS, Santo Domingo, República Dominicana. Diciembre 2020.

Toro, Jonathan (2021): Los países que adelantan reformas en sus impuestos para compensar el hueco fiscal. Periódico La República. Sección Hacienda. Colombia, 9 de abril del 2021. https://www.larepublica.co/globoeconomia/los-paises-que-adelantan-reformas-en-susimpuestos-para-compensar-el-hueco-fiscal-3154835. En línea al 5 de junio del 2021.

Ula, Moore (2020): Medidas Frente al Covid-19 en la República Dominicana. Revista Moore. Noviembre del 2020. https://www.moore.do/MediaLibsAndFiles/media/do.moorestephens.com/Documents/M-ULANEWSLETTER-MEDIDAS-FRENTE-AL-COVID-19-EN-RD-Noviembre-2020.pdf?ext=.pdf. En línea al 31 de mayo del 2021.

Velásquez, Melissa (2021): ¿Qué está pasando en Colombia? Reforma tributaria, protestas, militarización de ciudades y amenazas a la ONU. Publicado en CNN en Español. Fecha 5 de mayo del 2021. https://cnnespanol.cnn.com/2021/05/05/protestas-colombia-reformatributaria-violentas-militarizacion-ciudades-amenazas-a-la-onu-orix/.En línea al 21 de mayo 2021.

Zatsman, Ariel y Zentner, Joaquín (2021): República Dominicana: Aumentar los ingresos fiscales es clave para mejorar la calidad de vida de los ciudadanos. Banco Interamericano de Desarrollo (BID). April 20, 2021 April 20, 2021. 